L. Bădescu

Nagoya Math. J.

Vol. 71 (1978), 169-179

\title{
A REMARK ON THE GROTHENDIECK-LEFSCHETZ THEOREM ABOUT THE PICARD GROUP
}

\author{
LUCIAN BǍDESCU
}

Let $K$ be an algebraically closed field of arbitrary characteristic. The term "variety" always means here an irreducible algebraic variety over $K$. The notations and the terminology are borrowed in general from EGA [4].

Let $X$ be a projective non-singular variety embedded in the projective space $P^{n}=P$, and let $Y$ be a closed subvariety. Throughout this note we shall assume that $\operatorname{dim}(Y) \geqslant 2$ and that $Y$ is a scheme-theoretic complete intersection of $X$ with some hypersurfaces $H_{1}, \cdots, H_{r}$ of $P$, where $r=\operatorname{codim}_{X}(Y)$. Sometimes we shall simply say that $Y$ is complete intersection in $X$.

First of all recall the following result (see [5], [7]):

THEOREM A (Grothendieck-Lefschetz). In the above hypotheses, assume moreover that $K$ is the complex field and that $Y$ is non-singular of dimension $\geqslant 3$. Then the natural homomorphism of restriction of Picard groups

$$
\operatorname{Pic}(X) \rightarrow \operatorname{Pic}(Y)
$$

is an isomorphism.

Note. There is in fact a more precise statement than the above theorem, asserting that even the corresponding morphism between Picard schemes $\mathrm{Pic}(X) \rightarrow \underline{\mathrm{Pic}}(Y)$ is an isomorphism.

The above theorem implies in particular that the following homomorphisms of restriction are also isomorphisms (the hypotheses being the same as in theorem A):

$$
\begin{aligned}
\operatorname{Pic}(X) / Z\left[O_{X}(1)\right] & \rightarrow \operatorname{Pic}(Y) / Z\left[O_{Y}(1)\right] \\
\operatorname{Pic}^{\tau}(X) & \rightarrow \operatorname{Pic}^{\tau}(Y)
\end{aligned}
$$

Received August 9, 1977. 
where $Z\left[O_{X}(1)\right]$ is the subgroup of $\operatorname{Pic}(X)$ generated by the class $\left[O_{X}(1)\right]$ of the invertible sheaf $O_{X}(1)$ associated with the hyperplane section of $X$ via the embedding $X \subset P, O_{Y}(1)$ is the restriction to $Y$ of $O_{X}(1), \operatorname{Pic}^{\tau}(X)$ (resp. $\operatorname{Pic}^{0}(X)$ ) is the subgroup of $\operatorname{Pic}(X)$ consisting of all classes of invertible sheaves numerically (resp. algebraically) equivalent to zero (see [9]), and $N S(X)=\operatorname{Pic}(X) / \operatorname{Pic}^{0}(X)$ is the Néron-Severi group of $X$.

This result is no longer true in general if $Y$ is a surface (take for instance $P^{3}$ as $X$ and $P$, and any non-singular quadric or cubic surface in $P^{3}$ as $Y$ ). However one knows the following result (see [3]):

THEOREM B. Let $Y$ be a non-singular surface which is complete intersection in the projective space $P^{n}$ over an algebraically closed field of arbitrary characteristic. Then $\operatorname{Pic}^{\tau}(Y)=0$ and the class of $O_{Y}(1)$ is not divisible in $N S(Y)$.

The proof of theorem B given in [3] is a consequence of a careful study of the $\ell$-adic cohomology of $Y$ as well as of Hodge cohomology $H^{q}\left(Y, \Omega_{Y}^{p}\right)\left(\Omega_{Y}^{p}\right.$ denoting the sheaf of germs of algebraic differential forms of degree $p$ on $Y$ ). In other words theorem $\mathrm{B}$ asserts the following: $\operatorname{Pic}(Y)$ has no torsion and $\operatorname{Pic}(Y) / Z\left[O_{Y}(1)\right]$ has also no torsion.

The aim of this note is to find informations about the maps (1), (2), (3), (4) and (5) when $Y$ is a surface, complete intersection in $X$.

THEOREM. In the hypotheses stated at the beginning, assume moreover that $Y$ is a normal surface and the following condition is fulfilled:

$$
\text { (*) } \begin{array}{r}
H^{q}\left(X, O_{X}(m)\right)=0 \\
\text { for every } m<0 \text { and for every } 1 \leqslant q<\operatorname{dim}(X) .
\end{array}
$$

Then the maps (1),(2) and (5) are injective and have cokernels all isomorphic to the same group $E$, which is free of finite rank if char $(K)$ $=0$, and e-torsion-free of finite type if $\operatorname{char}(K)=p>0$, where $e$ is any positive integer prime to $p$. Moreover, the map (4) is always an isomorphism and the map (3) injective and even bijective if char $(K)$ $=0$.

Proof. It is based on the standard Lefschetz theory in 
Grothendieck's form (see [5], [7]), and on the theory of Picard schemes (see [6], [11]). We divide it into several steps.

Step 1. (Lefschetz theory). According with the notations and the context of [7], let $\hat{X}$ be the formal completion of $X$ along $Y$; by [7], chap. IV, theorem (1.5) and the proof of theorem (3.1), the map Pic $(X)$ $\rightarrow \operatorname{Pic}(Y)$ factorizes through $\operatorname{Pic}(X) \rightarrow \operatorname{Pic}(\hat{X}) \rightarrow \operatorname{Pic}(Y)$ and the map

$$
\operatorname{Pic}(X) \rightarrow \operatorname{Pic}(\hat{X})
$$

is an isomorphism.

On the other hand, if $Y_{n}=\left(Y, O_{X} / I^{n}\right)$ ( $I$ being the coherent sheaf of ideals of $Y$ in $X$ ), by a general statement (see loc. cit.) we have

$$
\operatorname{Pic}(\hat{X})=\operatorname{inv} \lim _{n} \operatorname{Pic}\left(Y_{n}\right)
$$

Step 2. Condition (*) implies that $H^{1}\left(Y, O_{Y}(m)\right)=0$ for every $m<0$.

Indeed, by induction on $r$ one can easily assume $r=1$, i.e. $Y$ $=X \cap H$ (scheme-theoretically), where $H$ is a hypersurface of $P$ of degree $d$ of equation $f=0$. We then get the exact sequence:

$$
0 \longrightarrow O_{X}(m-d) \stackrel{f}{\longrightarrow} O_{X}(m) \longrightarrow O_{Y}(m) \longrightarrow 0
$$

which induces the exact sequence of cohomology

$$
H^{q}\left(X, O_{X}(m)\right) \rightarrow H^{q}\left(Y, O_{Y}(m)\right) \rightarrow H^{q+1}\left(X, O_{X}(m-d)\right) .
$$

For $1 \leqslant q<\operatorname{dim}(Y)$ the first and the third cohomology group vanish, so that the middle one also vanishes.

Step 3. $H^{1}\left(Y, I^{n} / I^{n+1}\right)=0$ for every $n \geqslant 1$.

Indeed, because $Y$ is complete intersection of $X$ with the hypersurfaces $H_{1}, \cdots, H_{r}$ of degree $d_{1}, \cdots, d_{r}$, the conormal sheaf $I / I^{2}$ is isomorphic to $\oplus_{i=1}^{r} O_{Y}\left(-d_{i}\right)$ (see [7], page 106). Moreover, for every $n \geqslant 1$ the sheaf $I^{n} / I^{n+1}$ is isomorphic to the $n^{t h}$ symmetric power $S^{n}\left(I / I^{2}\right)$, and therefore is again a direct sum of line bundles of the form $O_{Y}(m)$ with $m<0$. So by step 2 we get the conclusion of step 3 .

Step 4. Consider the standard exact sequence (see [5], [7]) :

$$
0 \rightarrow I^{n} / I^{n+1} \rightarrow O_{Y_{n+1}}^{*} \rightarrow O_{Y_{n}}^{*} \rightarrow 1
$$


which induces the following exact sequence of cohomology:

$$
0 \stackrel{\text { by step } 3}{=} H^{1}\left(Y, I^{n} / I^{n+1}\right) \rightarrow \operatorname{Pic}\left(Y_{n+1}\right) \rightarrow \operatorname{Pic}\left(Y_{n}\right) \rightarrow H^{2}\left(Y, I^{n} / I^{n+1}\right)
$$

in which $H^{2}\left(Y, I^{n} / I^{n+1}\right)$ is a torsion-free group if $\operatorname{char}(K)=0$, and an $e$-torsion-free group if $p>0$, being in fact a $K$-vector space. Thus, one can identify $\operatorname{Pic}\left(Y_{n}\right)$ to a subgroup of $\operatorname{Pic}(Y)\left(=\operatorname{Pic}\left(Y_{1}\right)\right)$ in such a manner that $\left[O_{X}(1)\right]$ and $\left[O_{Y}(1)\right]$ become equal and the quotient group $\operatorname{Pic}\left(Y_{n}\right) / \operatorname{Pic}\left(Y_{n+1}\right)(n \geqslant 1)$ is :

-torsion-free if $\operatorname{char}(K)=0$,

- $e$-torsion-free if $p>0$, for any $e$ prime to $p$.

Then (6) and (7) from step 1 can be restated as follows:

$$
\operatorname{Pic}(X)=\bigcap_{n=1}^{\infty} \operatorname{Pic}\left(Y_{n}\right) \text {. }
$$

Now it is clear that (1) and (2) are injective maps and have the same cokernel $E$ which is:

-torsion-free if char $(K)=0$ (by (8) and the torsion-freeness of the groups Pic $\left(Y_{n}\right) / \operatorname{Pic}\left(Y_{n+1}\right)$ for every $\left.n \geqslant 1\right)$,

- $e$-torsion-free if $p>0$, where $e$ is any integer prime to $p$ (in the same way).

Step 5. For simplicity, let us set $p=1$ if $\operatorname{char}(K)=0$, and

$$
e \text {-Tors }(F)=\{x \in F / e x=0\} \quad \text { for every abelian group } F \text {. }
$$

Since Pic $(X) / \operatorname{Pic}^{\tau}(X)$ and $\operatorname{Pic}(Y) / \operatorname{Pic}^{\tau}(Y)$ have no torsion, one sees that in the commutative diagram

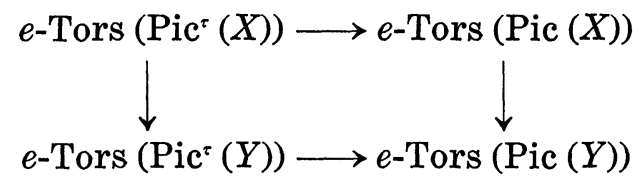

the horizontal arrows are isomorphisms, and, by step 4, the right vertical arrow is also an isomorphism, hence

$$
e \text {-Tors }\left(\operatorname{Pic}^{\tau}(X)\right) \cong e \text {-Tors }\left(\operatorname{Pic}^{\tau}(Y)\right)
$$

By a theorem of Matsusaka (see [9]) $\operatorname{Pic}^{r}(X) / \operatorname{Pic}^{0}(X)$ and $\operatorname{Pic}^{\tau}(Y) /$ $\mathrm{Pic}^{0}(Y)$ are finite groups. Considering the injective homomorphism

$$
e \text {-Tors }\left(\operatorname{Pic}^{0}(X)\right) \rightarrow e \text {-Tors }\left(\operatorname{Pic}^{0}(Y)\right) \text {. }
$$


I claim it has finite cokernel of cardinality at most $\lambda=\operatorname{order}\left(\operatorname{Pic}^{\tau}(X) /\right.$ $\left.\operatorname{Pic}^{0}(X)\right)$.

Indeed, consider the commutative diagram with exact lines

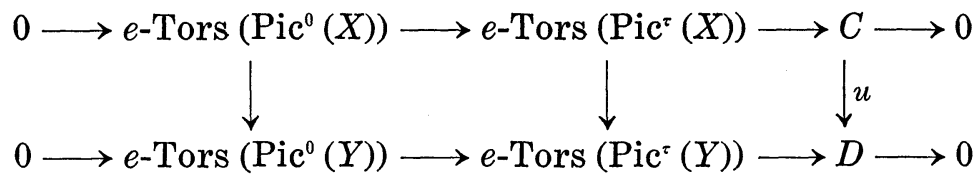

Taking into account of (9), one deduces that the cokernel of (10) is isomorphic to $\operatorname{Ker}(u)$. But the exact sequence (e-Tors is a left exact functor!)

$$
0 \rightarrow e \text {-Tors }\left(\operatorname{Pic}^{0}(X)\right) \rightarrow e \text {-Tors }\left(\operatorname{Pic}^{\tau}(X)\right) \rightarrow e \text {-Tors }\left(\operatorname{Pic}^{\tau}(X) / \operatorname{Pic}^{0}(X)\right)
$$

shows that $\operatorname{Ker}(u) \subset C \subset \operatorname{Pic}^{\tau}(X) / \operatorname{Pic}^{0}(X)$, hence the claim.

Step 6. Since $X$ is non-singular and $Y$ normal, the group-schemes $\mathrm{Pic}^{0}(X)$ and $\mathrm{Pic}^{0}(Y)$ are proper over $K$ (see [6], No. 236, theorem 2.1), therefore it makes sense to speak about the abelian varieties $\operatorname{Pic}^{0}(X)_{r e d}$ and $\operatorname{Pic}^{0}(Y)_{r e d}$. By [12] chap. II, §6, one gets :

$$
\text { order }\left(e-\operatorname{Tors}\left(\operatorname{Pic}^{0}(X)\right)\right)=e^{2 g}, \quad g=\operatorname{dim} \underline{\operatorname{Pic}}^{0}(X), e \text { prime to } p,
$$$$
\text { order }\left(e-\operatorname{Tors}\left(\operatorname{Pic}^{0}(Y)\right)\right)=e^{2 g^{\prime}}, \quad g^{\prime}=\operatorname{dim} \underline{\operatorname{Pic}}^{0}(Y) \text {. }
$$

By the injectivity of the map $\operatorname{Pic}^{0}(X) \rightarrow \underline{\operatorname{Pic}}^{0}(Y)$ and by step 5 we get the inequalities

$$
g \leqslant g^{\prime} \quad \text { and } \quad e^{2 g^{\prime}} \leqslant \lambda e^{2 g}
$$

Remarking that one can choose $e$ arbitrarily large (and prime to $p$ ), these inequalities imply $g=g^{\prime}$, i.e. the morphism $\underline{\operatorname{Pic}}^{0}(X) \rightarrow \underline{\operatorname{Pic}}^{0}(Y)$ is bijective, since it is injective and $\underline{\mathrm{Pic}}^{0}(X)$ and $\underline{\mathrm{Pic}}^{0}(Y)$ are abelian schemes of the same dimension. Consequently, we deduce that (4) is an isomorphism.

Step 7. The isomorphism (4), the injectivity of (1) and the $e$-torsion-freeness of $E$ show that (5) is also injective and that $N S(Y) / N S(X)$ (which can be identified with $E$ ) is e-torsion-free.

Step 8 (For char $(K)=0)$. By step 7 , Tors $(N S(X)) \cong \operatorname{Tors}(N S(Y))$, where if $F$ is an abelian group, Tors $(F)$ denotes the torsion subgroup of $F$. But taking into account of (4) and the equalities 
$\operatorname{Tors}(N S(X))=\operatorname{Pic}^{\tau}(X) / \operatorname{Pic}^{0}(X)$ and $\operatorname{Tors}(N S(Y))=\operatorname{Pic}^{\tau}(Y) / \operatorname{Pic}^{0}(Y)$ one concludes that (3) is an isomorphism.

Step 9. By Néron-Severi theorem for surfaces, $N S(Y)$ is finitely generated, hence $E$ is also finitely generated, since, by step 7, $E$ is a quotient of $N S(Y)$. If $\operatorname{char}(K)=0, E$ results therefore free of finite rank.

q.e.d.

COROLLARY 1. In the hypotheses stated at the beginning, assume that $Y$ is a normal surface and that the ground field is the complex field $\boldsymbol{C}$.

a) The maps (1), (2) and (5) are injective and yield the isomorphisms $\operatorname{Pic}(Y) \cong \operatorname{Pic}(X) \oplus Z^{s}, \operatorname{Pic}(Y) / Z\left[O_{Y}(1)\right] \cong \operatorname{Pic}(X) / Z\left[O_{X}(1)\right] \oplus Z^{s}$ and $N S(Y)$ $\cong N S(X) \oplus Z^{s}$, where $s$ is a non-negative integer.

b) The class of $O_{Y}(1)$ is not divisible in Pic $(Y)$ (resp. in $N S(Y)$ ) if and only if the class of $O_{X}(1)$ is not divisible in Pic $(X)(r e s p$. in $N S(X))$.

c) The morphism Pic $(X) \rightarrow$ Pic $(Y)$ between Picard schemes yields the isomorphisms $\operatorname{Pic}^{\tau}(X) \rightarrow \operatorname{Pic}^{\tau}(Y)$ and $\operatorname{Pic}^{0}(X) \rightarrow \operatorname{Pic}^{0}(Y)$, and in particular $\operatorname{dim} H^{1}\left(X, O_{X}\right)=\operatorname{dim} H^{1}\left(Y, O_{Y}\right)$.

Proof. First observe that condition (*) of the theorem is always fulfilled over the complex field, by Kodaira's vanishing theorem. a) is then a direct consequence of the theorem; b) results easily using the isomorphism

$$
\operatorname{Tors}\left(\operatorname{Pic}(X) / Z\left[O_{X}(1)\right]\right) \cong \operatorname{Tors}\left(\operatorname{Pic}(Y) / Z\left[O_{Y}(1)\right]\right)
$$

(resp. Tors $\left(N S(X) / Z\left\{O_{X}(1)\right\}\right) \cong$ Tors $\left.\left(N S(Y) / Z\left\{O_{Y}(1)\right\}\right)\right)$ combined with the injectivity of (1) (resp. of (5)). The proof of c) is contained in step 6 of the proof of the theorem, because an abelian scheme in characteristic zero is always reduced (this is a theorem of Cartier, see [11], lecture 25).

Remarks. 1. First of all recall the following theorem of Lefschetz about singular cohomology (see [1]) :

THEOREM C (Lefschetz). Assume that $Y$ is a non-singular complete intersection in the projective space $P^{n}=P$ over the complex field. Then the natural homomorphism 


$$
H^{q}(P, Z) \rightarrow H^{q}(Y, Z)
$$

induced by inclusion $Y \subset P$, is

(i) bijective for $q<d=\operatorname{dim}(Y)$, and

(ii) injective for $q=d$, and the quotient group $H^{d}(Y, Z) / H^{d}(P, Z)$ has no torsion.

As it is known (see [8]), theorem $\mathrm{A}$ can be deduced from part (i) of theorem $\mathrm{C}$, if we restrict ourselves to the case where $Y$ is a nonsingular complete intersection in $P^{n}$ (i.e. $X=P^{n}$ ) and $\operatorname{dim}(Y) \geqslant 3$. Proceeding analogously as in loc. cit., we shall see bellow that one can deduce from part (ii) of theorem $\mathrm{C}$ the following special case of corollary 1: if $Y$ is a non-singular surface which is complete intersection in $P^{n}=P$, then the map $\operatorname{Pic}(P) \rightarrow \operatorname{Pic}(Y)$ is injective and $\operatorname{Pic}(Y) / \operatorname{Pic}(P)$ has no torsion, i.e. theorem $\mathrm{B}$ for the complex field.

Indeed, using the exponential sequence for $P$ and $Y$, one gets the commutative diagram with exact lines:

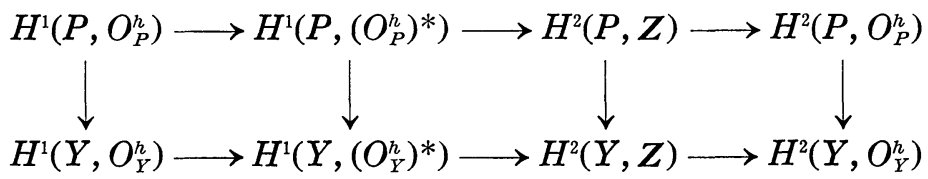

where $O^{h}$ denotes the sheaf of germs of holomorphic functions, and $\left(O^{h}\right)$ - the sheaf of germs of nowhere-vanishing holomorphic functions. By GAGA [16] and FAC [15], $H^{1}\left(P, O_{P}^{h}\right)=H^{2}\left(P, O_{P}^{h}\right)=H^{1}\left(Y, O_{Y}^{h}\right)=0$, $\operatorname{Pic}(P)=H^{1}\left(P,\left(O_{P}^{h}\right)^{*}\right)$ and $\operatorname{Pic}(Y)=H^{1}\left(Y,\left(O_{Y}^{h}\right)^{*}\right)$. Hence the map Pic $(P)$ $\rightarrow$ Pic $(Y)$ is injective since by (ii) of theorem $\mathrm{C}$ the map $H^{2}(P, Z)$ $\rightarrow H^{2}(Y, Z)$ is so, and $\operatorname{Pic}(Y) / \operatorname{Pic}(P)$ may be identified to a subgroup of $H^{2}(Y, Z) / H^{2}(P, Z)$, and this last group has no torsion by (ii) again.

2. If we restrict ourselves to the complex case, we see that corollary 1 may be regarded as a more general statement than theorem $B$.

3. By Lefschetz's principle corollary 1 is also valid over any algebraically closed field of characteristic zero.

4. By corollary 1 and the above remark, the map (3) turns out to be an isomorphism, provided that $\operatorname{char}(K)=0$. We do not know if (3) is always an isomorphism in the assumptions of the theorem and $\operatorname{char}(K)>0$. However, the answer is affirmative if $Y$ is a non-singular surface, complete intersection in $P^{n}$, as shows theorem $\mathrm{B}$. 
COROLlaRY 2 (Kleiman [9]). It is sufficient to prove Néron-Severi's theorem for projective non-singular surfaces in order to deduce it for projective non-singular varieties of arbitrary dimension (with arbitrary $\operatorname{char}(K))$.

Proof. Let $X$ be a projective non-singular veriety; we have to prove that $N S(X)$ is finitely generated if we assume this for projective non-singular surfaces. Since Néron-Severi's theorem is trivial for curves, we may assume $\operatorname{dim}(X) \geqslant 3$. Choose a projective embedding $i: X \smile P^{n}$; by FAC [15], $\S 75$, theorem $3, H^{q}\left(X, O_{X}(m)\right)=0$ for $1 \leqslant q$ $<\operatorname{dim}(X)$, provided that $m$ sufficiently small. Therefore composing eventually $i$ with a Veronese embedding $v_{d}: P^{n} \longleftrightarrow P^{N}\left(N=\left(\begin{array}{c}n+d \\ n\end{array}\right)\right.$ -1) of sufficiently high degree $d$, we get another embedding which satisfies this time condition $(*)$ of the theorem. Hence, without loss of generality, we may assume that $i$ satisfies $(*)$. Let then $Y$ be the intersection of $X$ with a general linear subspace of $P^{n}$ of codimension $=\operatorname{dim}(X)-2$. By Bertini's theorem, $Y$ is a non-singular surface. Hence, by the theorem, $N S(X)$ can be identified to a subgroup of $N S(Y)$, which was assumed to be finitely generated, so that $N S(X)$ is also finitely generated.

Remark. Instead of the injectivity of (5), Kleiman used Hodge index theorem (see [11], lecture 18) to get the statement of corollary 2. Actually, Kleiman showed more, namely that Néron-Severi's theorem is true for every complete variety (which may have singularities), assuming it for projective non-singular surfaces, by using moreover Chow's lemma and the resolution of singularities for surfaces.

COROLLARY 3. Let $X$ be a projective non-singular and arithmetically Cohen-Macaulay subvariety of the projective space $P^{n}$ over an algebraically closed field of arbitrary characteristic, and let $Y$ be a normal surface which is a complete intersection of $X$ with some hypersurfaces of $P^{n}$. Then the same conclusions as in the theorem hold, and moreover $\operatorname{Pic}^{0}(X)$ and $\operatorname{Pic}^{0}(Y)$ are both trivial.

Proof. We have to observe that the arithmetic Cohen-Macaulay-ness of $X$ in $P^{n}$ implies that condition $(*)$ of the theorem is fulfilled on one 
hand, and that $Y$ is also arithmetically Cohen-Macaulay in $P^{n}$, on the other hand. In particular we get that $H^{1}\left(X, O_{X}\right)=H^{1}\left(Y, O_{Y}\right)=0$, hence $\operatorname{Pic}^{0}(X)$ and $\operatorname{Pic}^{0}(Y)$ are trivial. Hence we see that a direct proof of corollary 3 does not need the discussion from steps 5 and 6, i.e. does not need the theory of Picard schemes.

COROLlaRY 4. In the hypotheses of corollary 3, assume moreover that the characteristic of the ground field is zero and that the group $\operatorname{Pic}(X) / Z\left[O_{X}(1)\right]$ has no torsion. Then every integral curve on $Y$ whose sheaf of ideals in $Y$ is invertible and which is set-theoretic complete intersection of $Y$ with a hypersurface of $P^{n}$, is actually a scheme-theoretic complete intersection of $Y$ with a hypersurface of $P^{n}$.

Proof. Using corollary 3 and isomorphism (11), one gets that the group $\mathrm{Pic}(Y) / Z\left[O_{Y}(1)\right]$ has no torsion. Moreover, since $Y$ is arithmetically Cohen-Macaulay in $P^{n}$, the Serre homomorphism $\alpha(Y): S(Y)$ $\rightarrow \bigoplus_{s=0}^{\infty} \Gamma\left(Y, O_{Y}(s)\right.$ ) (see EGA II or FAC) is an isomorphism, where by $S(Y)$ we mean the homogeneous coordinates ring of $Y$ in $P^{n}$. Corollary 4 follows now from the following lemma of Robbiano (see [13]) :

LEMma. Let $V \subset P^{n}$ be a closed subvariety of $P^{n}$ such that the Serre map $\alpha(V)$, is an isomorphism and $\mathrm{Pic}(V) / Z\left[O_{V}(1)\right]$ has no torsion. Then every closed integral subscheme $D$ of $Y$ whose sheaf of ideals in $Y$ is invertible and which is set-theoretic complete intersection of $V$ with a hypersurface of $P^{n}$. is actually a scheme-theoretic complete intersection of $V$ with a hypersurface of $P^{n}$.

Proof. $D$ can be regarded as a Cartier divisor on $Y$; since $D$ is integral and set-theoretic complete intersection of $V$ with a hypersurface of $P^{n}$, there are two positive integers $\alpha$ and $\beta$ such that $O_{V}(\alpha D) \cong O_{V}(\beta)$ $\left(O_{V}(\alpha D)\right.$ denoting the invertible $O_{V}$-module associated with the divisor $\alpha D)$. The torsion-freeness of $\mathrm{Pic}(V) / Z\left[O_{V}(1)\right]$ shows that there is a positive integer $\gamma$ such that $O_{V}(D) \cong O_{V}(\gamma)$. Now the divisor $D$ corresponds to a section $f \in \Gamma\left(V, O_{V}(\gamma)\right)$, and since $\Gamma\left(V, O_{V}(\gamma)\right) \cong S(V)_{r}$, we see that $D$ is a complete intersection of $V$ with the hypersurface $H$ of equation $F=0$, where $F$ is an homogeneous form of degree $\gamma$ in $n+1$ variables representing $f$ in $S(V)$.

Remark. Corollary 4 extends a result of Robbiano (see [14]), which essentially is the special case of corollary 4 in which $X$ is moreover 
arithmetically factorial in $P^{n}$ (modulo the fact that in [14] the ground field is not assumed to be algebraically closed; but also in our case this restriction is not really necessary, if we look carefully at the direct proof of corollary 3). The arithmetic factoriality of $X$ in $P^{n}$ implies that $\operatorname{Pic}(X) / Z\left[O_{X}(1)\right]=0$ and that every hypersurface of $X$ is cut out by a hypersurface of $P^{n}$.

Before giving an example, let us mention an elementary interpretation of the group $\operatorname{Pic}(V) / Z\left[O_{V}(1)\right]$ associated with a projective embedding $V \subset P^{n}$ of a non-singular variety $V$, namely: it is canonically isomorphic to the divisor class group of the normal graded ring $\oplus_{s=0}^{\infty} \Gamma\left(V, O_{V}(s)\right)$ (see [2], [10]).

ExAmple. Let $X=P^{m} \times P^{m^{\prime}}$ and embed $X$ in the projective space $P^{n}$ by :

$$
\left(a_{0}, \cdots, a_{m} ; b_{0}, \cdots, b_{m^{\prime}}\right) \rightarrow\left(\cdots, a_{0}^{\alpha_{0}} \cdots a_{m}^{\alpha_{m}} \cdot b_{0}^{\beta_{0}} \cdots b_{m^{\prime}}^{\beta_{m^{\prime}}}, \cdots\right)
$$

with $\alpha_{0}+\cdots+\alpha_{m}=s, \beta_{0}+\cdots+\beta_{m^{\prime}}=t, \alpha_{i} \geqslant 0, \beta_{j} \geqslant 0, s>0, t>0$ and $n=\left(\begin{array}{c}m+s \\ m\end{array}\right) \cdot\left(\begin{array}{c}m^{\prime}+t \\ m^{\prime}\end{array}\right)-1$. This embedding is always Cohen-Macaulay, and by above interpretation, $\operatorname{Pic}(X) / Z\left[O_{X}(1)\right] \cong Z \oplus Z / d Z$, where $d$ is the greatest common divisor of $s$ and $t$ (since $O_{X}(1) \cong p_{1}^{*} O(s) \otimes p_{2}^{*} O(t)$, where $p_{1}$ and $p_{2}$ are the canonical projections of our product). Therefore, if $m+m^{\prime} \geqslant 3$ and $s$ and $t$ are relatively prime each other, this embedding satisfies the hypotheses of corollary 4.

We do not know in general if the restriction about the characteristic of $K$ to be zero is really necessary in corollary 4. However, theorem B and Robbiano's lemma above allow us to deduce the following result, which extends to arbitrary characteristic the result of Robbiano from [13]:

COROLLARY 5. Let $Y$ be a projective non-singular surface, complete intersection in the projective space $P^{n}$ over an algebraically closed field $K$ of arbitrary characteristic. Then every integral curve $D$ on $Y$ which is set-theoretic complete intersection of $Y$ with a hypersurface of $P^{n}$, is actually a complete intersection of $Y$ with a hypersurface of $P^{n}$, and hence $D$ is a complete intersection in $P^{n}$.

\section{REFERENCES}

[1] Andreotti, A. and Frankel, T., The Lefschetz theorem on hyperplane sections, An- 
nals Math., 69 (3) (1959), 731-717.

[2] Danilov, V. I., The divisor class group of the completion of a ring (russian), Mat. Sbornik, $77(119): 4$ (1968), 533-541.

[ 3 ] Deligne, P., Cohomologie des intersections complètes, Sém. Géom. Alg. du Bois Marie (SGA 7, II), Springer Lect. Notes Math., No. 340 (1973).

[4] Dieudonné, J. and Grothendieck, A., (EGA) Eléments de Géométrie Algébrique, cap. I-III, Publ. Math. IHES (1960-1961).

[5] Grothendieck, A., (SGA-2) Séminaire de Géométrie Algébrique, Bures sur Yvette (1962).

[6] — - Fondements de la géométrie algébrique, extraits du sém. Bourbaki 19571962, Secr. Math. Paris, no. 232, 236.

[ 7 ] Hartshorne, R., Ample subvarieties of algebraic varieties, Springer Lect. Notes Math., No. 156 (1970).

[8] - Equivalence relations on algebraic cycles and subvarieties of small codimension, Proceedings Symp. Pure Math., Vol. 29 (Arcata, 1974) (1975).

[ 9 ] Kleiman, S., Toward a numerical theory of ampleness, Annals Math., 84 (1966), 239-344.

[10] Mori, S., On affine cones associated with polarized varieties, Japanese J. Math., Vol. 1(2) (1975), 301-309.

[11] Mumford, D., Lectures on curves on an algebraic surface, Annals Math. Studies, No. 59, Princeton (1966).

[12] - Abelian varieties, Lect. Tata Inst., Bombay (1968).

[13] Robbiano, L., A problem of complete intersections, Nagoya Math. J., 52 (1973), 129-132.

[14] — Some properties of complete intersections in "good" projective varieties, Nagoya Math. J., 61 (1976), 103-111.

[15] Serre, J.P., (FAC) Faisceaux algébriques cohérents, Annals Math., 61 (1955), 197-278.

[16] —, (GAGA) Géométrie Algébrique et Géométrie Analytique, Ann. Inst. Fourier, 6 (1956), 1-42.

University of Bucharest, Dept. of Mathematics 